\title{
Optimizing sperm cryopreservation in great scallop (Pecten maximus)
}

\author{
Marc SUQUET $^{\text {a }}$, Florent MALO, Clémence GouRTAY, Dominique RATISKOL, Marion RIOBE, \\ Jacqueline LE GRAND, Isabelle QUEAU and Christian MINGANT \\ Ifremer, UMR 6539, PFOM Department, Station Expérimentale d'Argenton, 11 Presqu'île du Vivier, 29840 Argenton, France
}

Received 28 January 2016; Accepted 19 April 2016

\begin{abstract}
A basic cryopreservation protocol for great scallop sperm was recently published, with which thawed sperm survival remained limited to $25 \%$ of control values (Suquet et al. 2014). We designed here a set of five experiments to test ways of improving thawed sperm survival and storage by studying the effects of (1) cryoprotectant concentration; (2) sugar incorporation in the extender; (3) egg yolk addition to the extender; (4) sperm dilution rate; and (5) storage capacity of thawed sperm at $4{ }^{\circ} \mathrm{C}$. A sixth experiment was then performed to compare rearing performances of larvae produced using thawed sperm from the optimal protocol defined in the present study with those produced using fresh sperm. During the first experiment, a significantly higher percentage of motile spermatozoa was recorded after thawing when the treatment had included 15 or $20 \%$ polyethylene glycol (PEG). In the second experiment, no significant improvement in post-thaw sperm motility was recorded when trehalose or glucose was added to the extender. In the third experiment, adding 10 or $20 \%$ egg yolk to the extender significantly decreased the percentage of motile sperm. During the fourth experiment, significantly higher percentages of motile sperm were observed for dilution rates ranging from $3: 1$ to $1: 1$ than for higher dilution rates. In the fifth experiment, a significant decrease in the percentage of post-thawed sperm was observed for storage durations of 30 and $60 \mathrm{~min}$, relative to shorter durations. During the last experiment, similar rearing performances were observed between larvae produced using fresh and thawed sperm, up to 9 days post fertilization. In conclusion, the high survival of sperm recorded after thawing (67\% of the control level) and the high rearing performances of progenies obtained using thawed sperm validate the use of the present improved cryopreservation protocol for sperm cryobanking in great scallop.
\end{abstract}

Keywords: Pecten maximus / mollusc / sperm / cryopreservation / larval rearing

\section{Introduction}

Agriculture intensification and industrialization are threats to biodiversity, leading to genetic erosion in domesticated animals (FAO 2007). In vitro conservation of genetic resources using cryopreservation of gametes or embryos is a powerful tool for maintaining biodiversity. National cryobanks have been developed in 50 countries (Paiva et al. 2014). In France, the national cryobank was founded in 1999, preserving biological material of domestic animals, including aquatic species such as salmonids and oysters (Danchin-Burge et al. 2002). To be a reliable cost-effective tool, sperm cryobanking needs to be optimised by improving gamete survival after thawing and validating freezing techniques by assessing the rearing performances of progenies produced using thawed sperm.

Since the review published by Gwo (2000), the number of studies on mollusc sperm cryopreservation has increased, but these still mainly concern oyster species (Hassan

\footnotetext{
a Corresponding author: msuquet@ifremer.fr
}

et al. 2015; Liu et al. 2015a; Paredes 2015). Optimization of cryoprotectant (CPA) concentration must aim for a balance between ensuring sperm protection and moderating cell toxicity. For example, a higher fertilization capacity was recorded for Pacific oyster (Crassostrea gigas) sperm cryopreserved using $5 \%$ propylene glycol, compared with higher CPA concentrations (Dong et al. 2005a). Using a combination of a permeating cryoprotectant such as DMSO, methanol or propylene glycol and a non permeating one, such as polyethylene glycol, led to the highest post-thaw motilities in Pacific oyster sperm (Dong et al. 2005b). Sugars are commonly added to the extender to enhance sperm membrane fluidity and to reduce ice formation. The post-thaw motility of farmed blacklip abalone (Haliotis rubra) sperm was improved by adding glucose to DMSO, but not by adding sucrose or trehalose (Liu et al. 2015b). Trehalose alone has an efficient role in protecting Pacific oyster sperm (Adams et al. 2004). Furthermore in Japanese pearl oyster (Pinctada fucata martensii), the post-thaw percentage of motile sperm increased when fetal bovine serum was added to the extender (Kawamoto et al. 2007). 
Aspects of the practical management of sperm samples should also be defined when developing a cryopreservation protocol. The capacity of sperm to be stored after thawing has only been studied at room temperature in Japanese pearl oyster, where a rapid decrease of the percentage of motile sperm was shown with increasing storage duration (Kawamoto et al. 2007). Then, comparing the rearing performances of larvae produced using thawed sperm with those assessed on larvae sired with fresh sperm is generally considered as the ultimate validation of a cryopreservation protocol.

Recently, a basic cryopreservation protocol was defined for great scallop sperm, using a saline solution (DCSB4) as an extender and polyethylene glycol (PEG) as a CPA. However, the survival rate of the thawed sperm remained limited (Suquet et al. 2014). The aims of the present paper are to improve post thawing sperm motility by (1) studying the effects of PEG concentration; (2) evaluating the effects of sugar or egg yolk incorporation in the extender; (3) determining the best sperm dilution rate in the extender; and (4) measuring subsequent survival of post-thawed sperm when stored at $4{ }^{\circ} \mathrm{C}$. The rearing performances of scallop larvae produced using thawed sperm were then compared to those obtained using fresh sperm.

\section{Material and methods}

\subsection{Animals}

Adult great scallops (mean weight \pm SEM: $94.1 \pm 3.7 \mathrm{~g}$ ) were caught by divers in the bay of Brest (Finistère, France) during the natural spawning period (May to October; Paulet et al. 1988). The great scallops were then transferred to the Argenton experimental hatchery (Ifremer) and maintained in a $100-\mathrm{L}$ race-way $\left(17{ }^{\circ} \mathrm{C}\right.$, salinity $34 \%$ o $)$ where they were fed daily on a mixture of microalgae (Isochrysis galbana and Chaetoceros calcitrans, $10^{9}$ cells of each species/day/animal) for one month. The sperm collection method and laboratory analysis are only given in brief below as these were previously described in detail in Suquet et al. (2014).

\subsection{Sperm collection and cryopreservation}

The great scallops were treated with $100 \mu \mathrm{l}$ of a $10 \mathrm{mM}$ serotonin solution, diluted in seawater (SW: $19{ }^{\circ} \mathrm{C}, 1 \mu \mathrm{m}$ filtered) and injected in the testes. They were then transferred to 2-L beakers filled with 0.5 -L SW. Sperm shedding was observed at 10 to $30 \mathrm{~min}$ post injection and samples were pipetted directly from the sperm plume, to ensure a high concentration. Sperm samples were then stored at $4{ }^{\circ} \mathrm{C}$ until use.

Sperm samples were individually processed $(n=5$ great scallops for each experiment). Unless stated below, sperm samples were diluted $1: 3$ with a solution containing the saline solution DCSB4 as an extender (Paniagua-Chavez et al. 1998) and polyethylene glycol (PEG: 20\%) as a cryoprotectant ( 3 straws for each treatment, maintained at $4{ }^{\circ} \mathrm{C}$ ). Straws $(500 \mu \mathrm{l})$ were cryopreserved on a tray floating $5.5 \mathrm{~cm}$ above liquid nitrogen (LN) for $10 \mathrm{~min}$ and then plunged into $\mathrm{LN}$. The straws were then thawed in a water bath $\left(25^{\circ} \mathrm{C}, 15 \mathrm{~s}\right)$.
Immediately post thawing, sperm motility was assessed after $1: 20$ to $1: 200$ dilution in the activating medium (SW, $5 \mathrm{~g} \mathrm{~L}^{-1}$ BSA, Tris $20 \mathrm{mM}$, adjusted to $\mathrm{pH} 8.1$ ), depending on the individual sperm concentration. Sperm samples of $7 \mu \mathrm{l}$ were transferred to Fast-Read 102 cells (Biosigma $囚$, Cona, Italy) and sperm motility was observed under a phase contrast microscope (Olympus BX51, ×20 magnification). The percentage of motile spermatozoa was visually estimated by assessing the numbers of motile cells in 6 samples $(n>30$ spermatozoa for each sample).

\subsection{Experimental design}

A set of five cryopreservation experiments was carried out in order to examine ways of improving post-thawing sperm survival, as measured by the percentage of motile spermatozoa. A sixth experiment was then done to compare the rearing performances of larvae produced using fresh and thawed sperm, cryopreserved with the optimal protocol defined by the preceding experiments.

The first experiment examined the effect of CPA concentration on sperm motility. Four concentrations of PEG $(5,10$, 15 and $20 \%$ ) and a combination of $10 \%$ PEG $+10 \%$ DMSO were tested.

In the second experiment, the effects of sugar addition to the extender were evaluated, using six experimental conditions: PEG 20\%, trehalose $500 \mathrm{mM}$, PEG 20\% + $100 \mathrm{mM}$ trehalose, PEG 20\% + $500 \mathrm{mM}$ trehalose, PEG 20\% + $100 \mathrm{mM}$ glucose and PEG 20\%+500 mM glucose. In the third experiment, the effects of egg yolk addition $(0,10$ or $20 \%)$ to the extender were examined.

In the fourth experiment, the effects of sperm dilution rate were assessed. Five dilution rates [sperm : (extender + CPA)] were examined $3: 1,2: 1,1: 1,1: 3$, and $1: 9$. In order to avoid confounding effects due to possible CPA toxicity, the volumes of CPA were maintained constant for all dilutions.

The fifth experiment examined the storage capacity of thawed sperm, i.e. sperm ability to maintain its movement when stored at $4{ }^{\circ} \mathrm{C}$, under three types of conditions: sperm without further dilution, sperm diluted (v/v) in SW and sperm diluted $(\mathrm{v} / \mathrm{v})$ in a medium mimicking great scallop seminal fluid (MMSF: SW diluted to 31\%o adding distilled water, $20 \mathrm{mM}$ Tris and $\mathrm{pH}$ adjusted to 6.4). To define the composition of MMFS, mean values of osmotic pressure $(831 \pm$ $\left.34 \operatorname{mosm~}^{-1}\right)$ and $\mathrm{pH}(6.40 \pm 0.05)$ were measured in the seminal fluid of scallops $(n=6)$. Under these conditions, sperm motility was assessed at 2, 10, 30 and 60 min after thawing.

The last experiment then assessed the rearing performances of larvae produced using thawed sperm, compared with those obtained using fresh sperm. Sperm of five great scallops was collected individually and each sample was separated into two portions of equivalent size: the first portion was stored at $4{ }^{\circ} \mathrm{C}$ until use and the second was cryopreserved using the best conditions defined in the present study [dilution rate $3: 1$, sperm: (extender $+\mathrm{CPA})]$, using 5 straws for each scallop. Eggs were obtained by thermal shock (from 18 to $23{ }^{\circ} \mathrm{C}$ ). To decrease self fertilization, eggs were filtered $(20 \mu \mathrm{m})$ and washed immediately post shedding. Eggs collected from two females were pooled and counted after transfer 


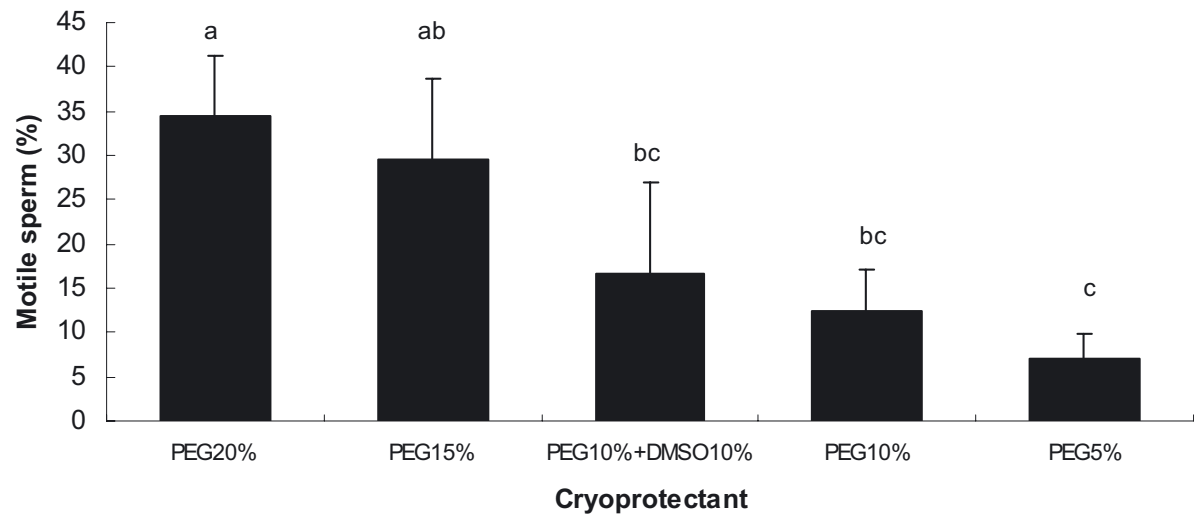

Fig. 1. Effect of cryoprotectant (single or combined) on the percentage of motile post-thaw spermatozoa $(n=5$ great scallops, different letters indicate significantly different results).

to a 2 -L cylinder (3 samples of $50 \mu \mathrm{l}$ each). The eggs were fertilized following a standardized protocol previously defined by Song et al. (2009) and adapted for great scallop: triplicate batches of 25000 eggs were each fertilized in $50 \mathrm{ml} \mathrm{SW}$, using a sperm-to-egg ratio close to 100, (as observed under the phase contrast microscope) for fresh and thawed sperm. After $45 \mathrm{~min}$ of contact between gametes, the beakers were filled with 2-L SW (total of 30 beakers: 5 great scallops $\times$ fresh or thawed sperm $\times$ triplicate). To estimate self-fertilization, triplicate samples of the egg pool were also incubated without sperm addition. Two days post fertilization, larvae were filtered $(40 \mu \mathrm{m})$, transferred to a $10 \mathrm{ml} \mathrm{SW}$ cylinder and counted (3 samples of $50 \mu \mathrm{l}$ each). The D-larval yield was estimated: D-larval yield day $2=$ [(number of D-larvae - number of self fertilized D-larvae)/(25000-number of self fertilized D-larvae) $\times 100$. Then, larvae were reared in 2 -L beakers: each second day, they were filtered $(40 \mu \mathrm{m})$ and the SW was changed. Larvae were fed on a mixture of Isochrysis galbana, Chaetoceros calcitrans and Skeletonema marinoï (15 algae of each species $\mu 1^{-1}$ ). Erythromycin was also added (for each beaker, $4 \mathrm{ml}$ of a solution composed of $2 \mathrm{~g}$ antibiotic diluted in 1-L SW). Larval survival was assessed nine days post fertilization: D-larval yield day $9=$ (number of larvae at day $9 /$ number of larvae at day 2) $\times 100$. Furthermore, D-larval length and circularity were estimated by image analysis (using LAS and Image J, respectively; 30 larvae measured for each experimental condition).

\subsection{Statistical analysis}

Results are presented as mean \pm SEM. The percentage of motile sperm and D-larval yields were arcsin square root transformed and their means compared using paired Student $t$-tests and one- or two way-ANOVA. For ANOVA and when differences were significant, a Fisher a posteriori test was used for mean comparisons.

\section{Results}

For the first five experiments, the mean percentage of motile fresh sperm was $87.2 \pm 1.5 \%$. During the first
Table 1. Rearing performances of larvae produced using fresh or thawed sperm ( $p$ indicates within line comparison).

\begin{tabular}{cccc}
\hline Parameter & Fresh & Thawed & $p$ \\
\hline D-larval yield at day 2 (\%) & $9.7 \pm 3.3$ & $3.0 \pm 1.0$ & 0.064 \\
D-larval yield at day 9 (\%) & $64.8 \pm 7.5$ & $57.1 \pm 2.9$ & 0.338 \\
D-larval length (mm) & $0.144 \pm 0.003$ & $0.149 \pm 0.001$ & 0.139 \\
D-larval circularity & $0.626 \pm 0.049$ & $0.709 \pm 0.073$ & 0.961 \\
\hline
\end{tabular}

experiment, a significantly higher percentage of motile spermatozoa was recorded post-thawing when 15 or $20 \%$ PEG had been used ( $p=0.027$, Fig. 1$)$, compared with the other CPA treatments. Using a combination of a permeating and a non permeating CPA did not improve post-thawing sperm motility. In the second experiment, no significant improvement in the post-thaw sperm motility was shown following the addition of trehalose or glucose to the extender, whatever sugar concentration used. Furthermore, no significant differences were recorded, using $500 \mathrm{mM}$ trehalose as a single CPA $(p=0.278$; $20 \%$ PEG $+100 \mathrm{mM}$ trehalose: $38.6 \pm 5.2 \%$ motile sperm, $20 \%$ PEG: $32.7 \pm 5.2 \%, 20 \%$ PEG + $500 \mathrm{mM}$ glucose: $30.6 \pm 4.5 \%, 20 \%$ PEG $+500 \mathrm{mM}$ trehalose: $27.5 \pm 4.7 \%$, $20 \%$ PEG + $100 \mathrm{mM}$ glucose: $23.7 \pm 4.7 \%, 500 \mathrm{mM}$ trehalose: $11.6 \pm 2.3 \%$ ). In the third experiment, adding 10 or $20 \%$ egg yolk to the extender significantly decreased the percentage of motile sperm post-thawing ( $p=0.001$, Fig. 2 ). During the fourth experiment, significantly higher percentages of motile sperm were observed for dilution rates [sperm: (extender + CPA)] ranging from $3: 1$ to $1: 1(p=0.032$, Fig. 3$)$ than for more diluted treatments. In the fifth experiment and after sperm dilution in the activating medium, a significant decrease of the motility percentage of post-thawed spermatozoa was observed for storage durations of 30 and $60 \mathrm{~min}$ at $4{ }^{\circ} \mathrm{C}$, relative to shorter periods ( $p=0.001$, Fig. $4 \mathrm{~A})$. Furthermore, a significantly higher percentage of motile spermatozoa was observed for sperm samples diluted (v/v) in SW or in a medium mimicking great scallop seminal fluid ( $p=0.002$, Fig. 4B), compared to undiluted samples.

During the last experiment, no significant difference in rearing performances were observed between larvae produced using fresh or thawed sperm, including survival rates at day 2 and 9, larval length and circularity (Table 1). 


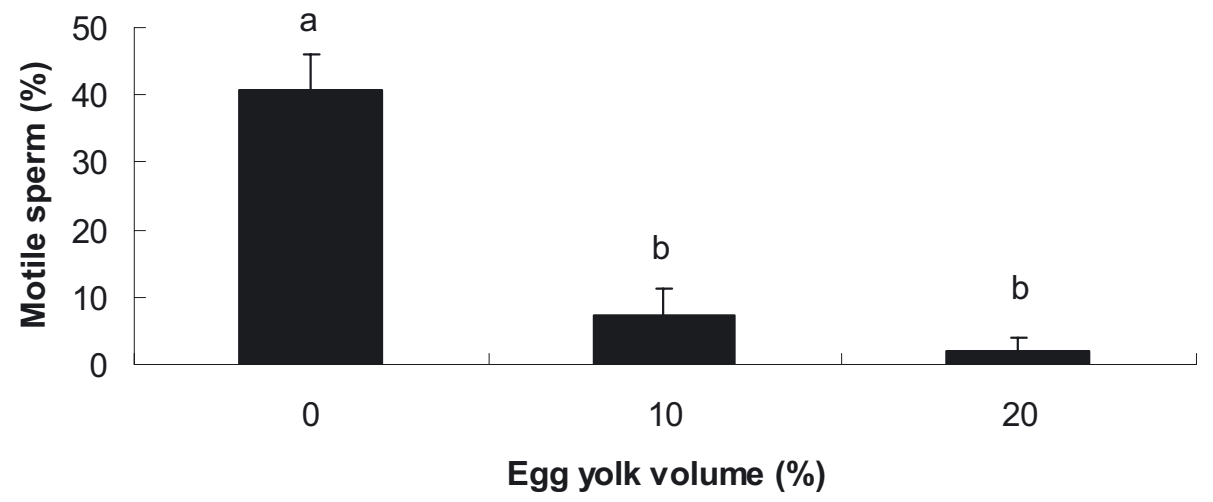

Fig. 2. Effect of egg yolk addition to the extender on the percentage of motile post-thaw spermatozoa $(n=5$ great scallops, different letters indicate significantly different results).

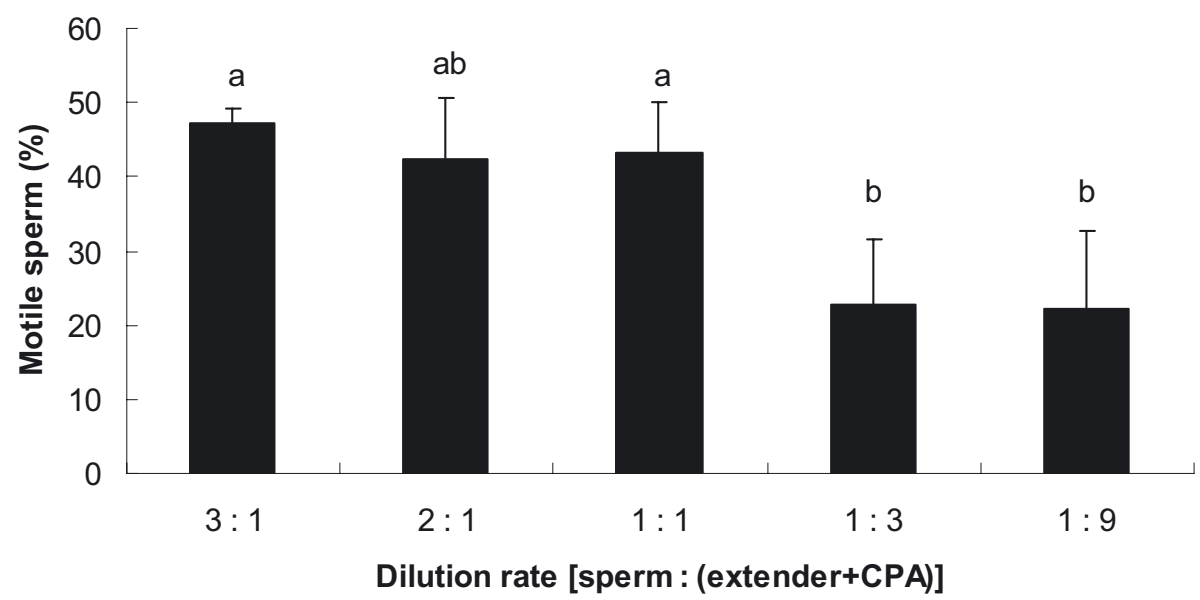

Fig. 3. Effect of sperm dilution rate (in the extender + CPA) on the percentage of motile post-thaw spermatozoa $(n=5$ great scallops, different letters indicate significantly different results).

\section{Discussion}

A basic cryopreservation protocol previously published for great scallop sperm (Suquet et al. 2014) led to limited postthawing survival of $25 \pm 2 \%$ (calculated as a percentage of the percentage of motile sperm from a fresh control), whereas the modifications adopted in the conclusion of the present study improve post-thawing sperm survival to $67 \pm 5 \%$. The present improvement was mainly due to the optimisation of CPA concentration and sperm dilution rate in the extender.

The first key element was the choice of PEG concentration. PEG has rarely been used as a CPA for invertebrate sperm; $70 \%$ of the studies published on marine species used DMSO as the most successful CPA out of those they tested (Paredes 2015). Furthermore, PEG was only used in one of the 23 studies reviewed by Hassan et al. (2015), where it was used in sperm cryopreservation in oyster species. Like sugars, proteins and polymers, PEG belongs to the non-permeable CPAs, which have been found to favour influx and efflux of solutes during the freezing process by improving sperm membrane stability (Meryman 1971). In Pacific oyster, the percentage of motile post-thawed sperm was not significantly different between 5 and 10\% PEG (Dong et al. 2006). PEG toxicity to gametes of aquatic species has rarely been studied but it showed low toxicity towards sea urchin (Paracentrotus lividus) sperm and no effect on egg fertilization capacity below a concentration of $200 \mathrm{mg} \mathrm{L}^{-1}$ (Ghirardini et al. 2001). This low toxicity to gametes could contribute to the high motility of thawed great scallop sperm recorded when high concentrations of PEG (15 or $20 \%$ ) were added. However, diluting thawed sperm in seawater or in a medium mimicking great scallop seminal fluid, improved sperm storage capacity, suggesting that PEG had a toxic effect, post thawing. In contrast to results found in the present study, combining a permeating CPA (DMSO: 6\%) with a non permeating one (PEG: 2\%) led to a higher post-thaw motility of Pacific oyster sperm than DMSO or PEG used as a single CPA (Dong et al. 2005b). However, higher CPA concentrations were used in the present work ( $10 \%$ for each CPA) than in this previous study.

The second key step of optimisation of the great scallop sperm cryopreservation protocol was the choice of sperm dilution rate in a medium composed of the extender and CPA. During the cryopreservation process, the effect of sperm dilution rate is species specific: in Japanese pearl oyster, increasing dilution ratio from $1: 2.5$ to $1: 50$ improved the percentage of motile thawed sperm (Kawamoto et al. 2007), suggesting a detrimental effect of high cell concentration during cryopreservation. This "packing effect", observed at high 



Fig. 4. Effect of post-thawing sperm storage duration (A) and post-thawing sperm dilution (B), on the percentage of motile spermatozoa $(n=5$ great scallops, different letters indicate significantly different results, MMSF: medium mimicking seminal fluid).

density, may be the result of harmful mechanical contact between cells (De Loecker et al. 1998). In Greenshell mussel (Perna canaliculus), however, sperm diluted $1: 1$ had a 10 fold higher fertilization capacity, post thawing, compared with samples diluted 1:3 (Smith et al. 2012). In agreement with our observations on great scallop, this detrimental effect of sperm dilution suggests that some components of the seminal fluid of these species are involved in sperm protection, as reported in fish species (Ciereszko 2008). In great scallop, it seems unlikely that proteins play this protecting role during sperm cryopreservation, because the addition of 10 or $20 \%$ egg yolk (from hen's eggs) decreased the motility of post-thawed spermatozoa in the present study. However, egg yolk contains also lipids, and proteins required for scallop sperm could be different from those included in this chemical. In contrast, adding 10 or $20 \%$ fetal bovine serum has a protective effect during the cryopreservation of Japanese pearl oyster sperm (Kawamoto et al. 2007).

Addition of sugar supplements to the extender has shown positive effects in Greenshell mussel sperm when associated with DMSO as a permeable CPA (Smith et al. 2012), while no effects were observed on blue mussel (Mytilus galloprovincialis) sperm when they were combined with the same permeable CPA (Liu et al. 2015c). In the present study, no improvement in the post-thaw sperm motility was revealed by the addition of trehalose or glucose to the extender, when associated with PEG, a non-permeable CPA. Adding sugars to the extender may play several roles: (i) improving sperm resistance to cryopreservation by stabilizing sperm membranes and decreasing intracellular ice formation; (ii) decreasing permeable CPA toxicity by its substitution with sugars; (iii) supplying an energy source sustaining sperm movement (Hassan et al. 2015; Paredes 2015). Further descriptive studies are required to understand the complex role of sugar in the extenders.

In great scallop, similar rearing performances were found here between larvae produced using fresh or thawed sperm, up to 9 days post fertilization. The survival of Pacific oyster progenies produced using fresh and thawed sperm and their larval growth were not significantly different up to 50 days post fertilization (Zhang et al. 2012). The rearing performances of oysters (Crassostrea tulipa), assessed up to 16 days post fertilization, were not affected by sperm cryopreservation (Yankson and Moyse 1991). The present results confirm the high quality of thawed great scallop sperm as previously assessed using a panel of biomarkers (Suquet et al. 2016). Finally, the low 
D-larval yield obtained using fresh sperm (9.3\%) confirm the limited control of scallop reproduction and larval rearing, as previously suggested by Robert and Gerard (1999).

In conclusion, great scallop sperm can be cryopreserved using the following conditions: DCSB4 as an extender, 20\% PEG as a CPA, $3: 1$ sperm dilution rate [sperm : (extender + CPA)], straws cryopreserved $5.5 \mathrm{~cm}$ above LN, sperm thawing at $25^{\circ} \mathrm{C}$, immediate dilution in seawater of thawed sperm (1:1), sperm storage at $4{ }^{\circ} \mathrm{C}$ and sperm use within 30 min post thawing. The high rearing performances of larvae produced using thawed sperm validate the use of the present cryopreservation protocol for sperm cryobanking in great scallop.

Acknowledgements. The authors would like to thank Stephane Pouvreau (Ifremer) and Matthias Huber (Ifremer) for fishing the scallops and Marie Louise Muzellec and Florian Breton (écloserie du Tinduff) for technical advice on great scallop spawning. Many thanks to Helen McCombie for corrections of the English. Financial support was provided by the CRB-Anim infrastructure project, ANR-11INBS-0003, funded by the French National Research Agency in the frame of the "Investment for the Future program".

\section{References}

Adams S.L., Smith J.F., Roberts R.D., Janke A.R., Kaspar H.F., Tervit H.B., Pugh P.A., Webb S.C., King N.G., 2004, Cryopreservation of sperm of the Pacific oyster (Crassostrea gigas): development of a practical method for commercial spat production. Aquaculture 242, 271-282.

Ciereszko A., 2008, Chemical composition of seminal plasma and its physiological relationship with sperm motility, fertilizing capacity and cryopreservation in fish. In: Alavi S.M.H., Cosson J., Coward K., Rafiee G. (Eds.), Fish spermatology, Oxford, Alpha Science International Ltd, pp. 215-240.

Danchin-Burge C., Bibe B., Planchenault D., 2002, The French national cryobank: creation of a cryogenic collection for domestic animal species. Institut de l'élevage, September 2002, 1-4.

De Loecker W., Koptelov V.A., Grishenko V.I., De Loecker P., 1998, Effects of cell concentration on viability and metabolic activity during cryopreservation. Cryobiology 37, 103-109.

Dong Q., Eudeline B., Huang C., Allen S.K.A., Tiersch T., 2005a, Commercial-scale sperm cryopreservation of diploid and tetraploid Pacific oysters, Crassostrea gigas. Cryobiology 50, $1-$ 16.

Dong Q., Huang C., Eudeline B., Tiersch T., 2005b, Systematic factor optimization for cryopreservation of shipped sperm samples of diploid Pacific oyster, Crassostrea gigas. Cryobiology 51, 176195.

Dong Q., Huang C., Eudeline B., Allen S.K. Jr, Tiersch T., 2006, Systematic factor optimization for sperm cryopreservation of tetraploid Pacific oyster, Crassostrea gigas. Theriogenology 66, 387-403.

FAO, 2007, The state of the world's animal genetic resources for food and agriculture- in brief. Commission on genetic resources for food and agriculture. Rome.

Ghirardini A.V., Novelli A.A., Likar B., Pojana G., Ghetti P.F., Marcomini A., 2001, Sperm cell toxicity test using sea urchin (Paracentrotus lividus) Lamarck (Echinodermata: Echinoidea) sensitivity and discriminatory ability toward anionic and nonionic surfactants. Env. Tox. Chem. 20, 644-651.
Gwo J.C., 2000, Cryopreservation of aquatic invertebrate semen: a review. Aquac. Res. 31, 259-271.

Hassan M.M., Qin J.G., Li, X., 2015, Sperm cryopreservation in oysters: A review of its current status and potentials for future application in aquaculture. Aquaculture 438, 24-32.

Kawamoto T., Narita T., Isowa K., Aoki H., Hayashi M., Komaru A., Ohta H., 2007, Effects of cryopreservation methods on post-thaw motility of spermatozoa from the Japanese pearl oyster, Pinctada fucata martensi. Cryobiology 54, 19-26.

Liu Y., Li X., Robinson N., Qin J., 2015a, Sperm cryopreservation in marine mollusk : a review. Aquacult. Int. 23, 1505-1524.

Liu Y., Xu T., Robinson N., Qin J., Li, X., 2015b, Cryopreservation of sperm in farmed blacklip abalone (Haliotis rubra Leach, 1814). Aquac. Res. 46, 2628-2636.

Liu B., Liu Y., Liu S., Xu T., Liu Q., Liu X., 2015c, Cryopreservation of strip spawned sperm using non-programmable freezing technique in the blue mussel Mytilus galloprovincialis. Aquac. Res. $1-11$.

Meryman H.T., 1971, Cryoprotective agents. Cryobiology 8, $173-$ 183.

Paiva S.R., McManus C., Blackburn H., 2014, Conservation of animal genetic resources: the next decade. Proc. 10th world congress of genetics applied to livestock production, Vancouver, Canada, 17-22 August 2014. https://asas.org/docs/default-source/ wcgalp-proceedings-oral/029_paper_10327_manuscript_1320_ 0. df? sfvrsn=2.

Paniagua-Chavez C.G., Buchanan J.T., Tiersch T., 1998, Effects of extender solutions and dilution on motility and fertilizing ability of Eastern oyster sperm. J. Shellfish Res. 17, 231-237.

Paredes E., 2015, Exploring the evolution of marine invertebrate cryopreservation - Landmarks, state of the art and future lines of research. Cryobiology 71, 198-209.

Paulet Y.M., Lucas A., Gerard A., 1988, Reproduction and larval development in two Pecten maximus (L.) populations from Brittany. J. Exp. Mar. Biol. Ecol. 119, 145-156.

Robert R., Gérard A., 1999. Bivalve hatchery technology: The current situation for the Pacific oyster Crassostrea gigas and the scallop Pecten maximus in France. Aquat. Living Resour. 12, 121-130.

Smith J.F., Adams S.L., Gale S.L., McGowan L.T., Tervit H.R., Roberts R.D., 2012, Cryopreservation of Greenshell mussel (Perna canaliculus) sperm. I. Establishment of freezing protocol. Aquaculture 334-337, 199-204.

Song Y.P., Suquet M., Quéau I., Lebrun L., 2009, Setting of a procedure for experimental fertilization of Pacific oyster (Crassostrea gigas) oocytes. Aquaculture 287, 311-314.

Suquet M., Arsenault-Pernet M.E.J., Ratiskol D., Mingant C., 2014, Cryopreservation of great scallop (Pecten maximus) sperm: effect of extender, cryoprotectant and cooling rate on sperm survival. Aquat. Living Resour. 27, 35-40.

Suquet M., Gourtay C., Donval A., Le Goïc N., Quere C., Malo F., Le Grand J., Ratiskol D., Mingant C., Fauvel C., 2016. The quality of great scallop (Pecten maximus) sperm after thawing. Gen. Comp. Endocrinol., in press.

Yankon K., Moyse J., 1991, Cryopreservation of the spermatozoa of Crassostrea tulipa and three other oysters. Aquaculture 97, 259267.

Zhang X., Li X., Clarke S., Li X., 2012, The development of Pacific oysters Crassostrea gigas produced using cryopreserved sperm. In: Qin J.G. (Ed.), Oysters: physiology, ecological distribution, and mortality, New York, Nova, pp. 1-18. 